\title{
On the MIMO Capacity for Distributed System under Composite Rayleigh/Rician Fading and Shadowing
}

\author{
Santiago González-Aurioles, ${ }^{1}$ J. L. Padilla, ${ }^{2}$ P. Padilla, ${ }^{3}$ \\ Juan F. Valenzuela-Valdés, ${ }^{1,3}$ and Juan C. Gonzalez-Maciass ${ }^{1}$ \\ ${ }^{1}$ Department of Computer and Telematics Systems Engineering, University of Extremadura, \\ Avenida Santa Teresa del Jornet No. 38, 06800 Merida, Spain \\ ${ }^{2}$ Nanolab, Ecole Polytechnique Fédérale de Lausanne, Lausanne, Switzerland \\ ${ }^{3}$ Department of Signal Theory, Telematics and Communications, CITIC, University of Granada, \\ C/Daniel Saucedo s/n, 18071 Granada, Spain \\ Correspondence should be addressed to Juan F. Valenzuela-Valdés; juanvalenzuela@unex.es
}

Received 1 September 2014; Revised 30 November 2014; Accepted 30 November 2014

Academic Editor: Bo Ai

Copyright (C) 2015 Santiago González-Aurioles et al. This is an open access article distributed under the Creative Commons Attribution License, which permits unrestricted use, distribution, and reproduction in any medium, provided the original work is properly cited.

\begin{abstract}
Wireless channels are commonly affected by short-term fading and long-term fading (shadowing). The shadowing effects must be taken into account also when mobility is present in the wireless scenario. Using a composite fading model, the total channel capacity can be studied for a scenario with short-term Rayleigh fading along with shadowing. This work provides quantitative results for these kinds of scenarios with Rayleigh fading and shadowing, considering also multiple-input and multiple-output systems, which have not been previously reported. In addition, the channel capacity has been studied in depth in its relation with the shadowing level, signal to noise ratio, and the number of elements in the multiple-input and multiple-output system. Moreover, the channel performance with shadowing has been compared to the one without it. Furthermore, Rician model with shadowing is studied and its results are reported. In addition, correlated and experimental results are provided. It is identified that the distributed MIMO systems can benefit from shadowing in Rician channels. This advantage has not been reported previously. This type of fading is proposed for massive MIMO by others and our results open the door to emulate massive MIMO on a reverberation chamber.
\end{abstract}

\section{Introduction}

Many theoretical and experimental studies have been carried out to predict the radio propagation characteristics. These studies aim to analyze propagation problems and propose statistical models for fading. Rayleigh, Rician, Nakagami-m, and Weibull are arguably the most popular statistical models for fading. All these fading models can be studied in scenarios on which the channel variations occur on a large scale period (i.e., variations due to turns in a corner, movements behind a large building, or the entrance to a building). This large-scale fading is often called shadowing.

This long-term energy variability in multipath fading channels is widely accepted to be properly described by lognormal statistics $[1,2]$. While the use of multiple antennas at the transmitter and receiver provides reasonable protection against short-term fading, it does not offer any kind of protection against shadowing, as the mitigation of shadowing requires the use of macrodiversity [3]. Macrodiversity implies the combination of signals from two or more base stations, being separated by a certain distance.

Cooperative networking has emerged as a core component of future wireless networks since it provides high data rate communication over large geographical areas. In this situation, distributed multiple-input multiple-output (DMIMO) communication arises as a promising tool for achieving large performance gains in wireless networks, similar to those provided by conventional MIMO in a point-to-point wireless channel. In these cases, wireless users with a single transmission and reception antenna cooperate in clusters to 
form distributed transmitting and receiving antenna arrays. A common assumption in the performance analysis of DMIMO systems is that channels between different pairs of nodes are subject to independent fading $[4,5]$. Analogous to the point-to-point case, this leads to capacity gains which are linear in the number of nodes $(M)$ contained in the transmission and reception clusters. In a distributed configuration, nodes (or antennas) are typically further away one from the other than for a classical MIMO one, so that an independent and identically distributed (i.i.d) fading model can be considered appropriate.

A D-MIMO system is able to combine the advantages of a point-to-point MIMO and a distributed antenna system (DAS) [6] and to have the ability to exploit spatial micro- and macrodiversity simultaneously [7-9]. For that reason, the dual effect of fading and shadowing, that is, the composite fading channel that addresses both macroscopic and microscopic fading, is gaining attention again [10-12]. A composite probability density function model is used in [10] for the outage analysis of a MIMO scheme operating in shadowed fading channels. Moreover, in [11] correlated shadowing is analyzed. Therefore, in this paper an analysis of D-MIMO capacity for both the microscopic fading (Rayleigh and Rician) channel and the composite channel is performed. With this model it is presented that the shadowing can improve the capacity in some conditions when composite fading is considered.

The rest of the document is organized as follows. Section 2 describes the models used and Section 3 validates these models. Sections 4 and 5 provide D-MIMO capacity results for Rayleigh shadowed fading and Rician shadowed fading, respectively. Finally, in Section 6 correlated and experimental results are shown, and the conclusions are outlined in Section 7.

\section{Composite Model for Microdiversity and Macrodiversity}

In this work, a wireless communication system that employs $T$ transmitting and $R$ receiving antennas is considered. In what follows, the analysis is restricted to the frequency flatfading case, with shadowing. It is assumed that the channel state information (CSI) is perfectly known by the receiver but is unknown by the transmitter and it is, therefore, unable to adapt its transmission strategy. At the receiver, the received signal $y$ is given by

$$
y=H x+n,
$$

where $x$ is the transmitted signal vector with total transmitted power $E\left(x x^{T}\right)=P$ and the terms of noise vector $n$ are modeled as zero-mean complex Gaussian random variables with variance $N_{0}=2$ per dimension and $H$ is the channel matrix that can be modeled as [13-15]

$$
H=\sqrt{s} x H_{\text {micro }}
$$

where $s$ is a log-normal random matrix representing the shadowing effects and $H_{\text {micro }}$ is a matrix representing the small-scale fading.
Furthermore, we assume that the shadowing value $s$ equally affects all the elements of $H_{\text {micro }}$ and $s$ is independent from $H_{\text {micro }}$. At this point, the generation of the $s$ matrix is [7]

$$
s=f_{\text {Nor }-L}(\mu, \sigma, R, T)+j * f_{\text {Nor-L }}(\mu, \sigma, R, T),
$$

where $f_{\text {Nor-L }}(\mu, \sigma, R, T)$ produces Gaussian random variables matrix with dimensions $R \times T$ with a log-normal distribution with mean equal to $\mu$ and variance equal to $\sigma$. The other term of $H$ is calculated for Rayleigh fading in the following way:

$$
H_{\text {micro_Ray }}=f_{\text {Nor }}(R, T)+j * f_{\text {Nor }}(R, T) \text {, }
$$

where $f_{\text {Nor }}(R, T)$ produces a random signal with dimensions $R \times T$ with mean equal to 0 and variance equal to 1 .

If it is desired to generate a channel matrix for Rician fading, the transformation developed by [16] may be applied to $H_{\text {micro_Ray. }}$ This transformation is applied in $[17,18]$ to obtain $H_{\text {micro_Rician }}$.

Thus, it is possible to generate the $H$ channel matrix with Rayleigh or Rician fading and lognormal shadowing for any parameter of shadowing and any number of elements using (2). Finally, the instantaneous channel capacity for MIMO systems can be calculated with the well-known expression defined in [19]:

$$
C=\log _{2}\left(\operatorname{det}\left[I_{R}+\frac{\mathrm{SNR}}{T} H * H^{\mathrm{T}}\right]\right),
$$

where det[] is the determinant of [], SNR is the signal to noise ratio, and $H^{\mathrm{T}}$ is the conjugate transpose of $H$. The system has $T$ antennas at the transmitter and $R$ antennas at the receiver, and $I_{R}$ is the identity matrix with dimensions $R \times R$. The ergodic (mean) capacity of the random MIMO channel is the mean over a variety of channel realizations of the ergodic fading process. In this paper we use 100000 realizations for each emulated point.

\section{Validation Model for Lognormal Distribution Matrix}

The equations for estimating the ergodic Shannon capacity of a log-normal channel have been recently presented in the literature $[20,21]$, and an interesting review of the state of the art and a new formula can be found in [22]. In this paper, the validation is carried out through a comparison of capacity results for a $1 \times 1$ scheme. Our simulation model is evaluated by comparing its results to those obtained using the model of [20]. Figure 1 shows that our simulation model matches properly the results of applying this model.

\section{Results for Composite Channel (Rayleigh + Shadowing)}

Most of the referred literature [10-12] and other existing literature are centered on the effect of macrodiversity and microdiversity on the average error probability. However, there is a lack of studies of both effects on MIMO capacity, and only a few results $[13-15,23]$ have been published. 


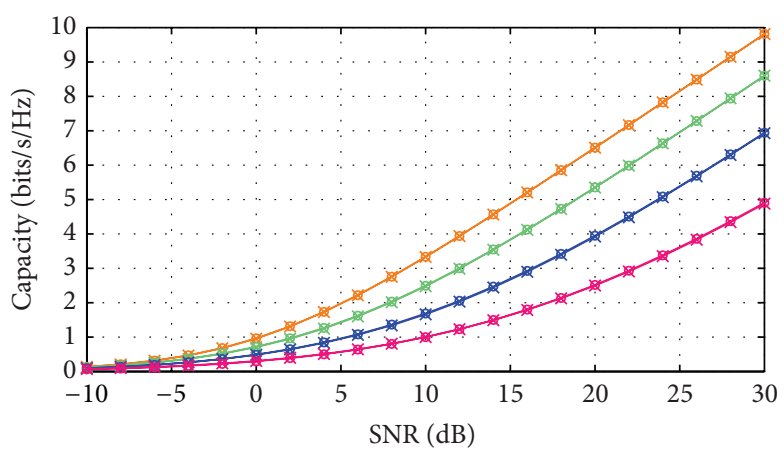

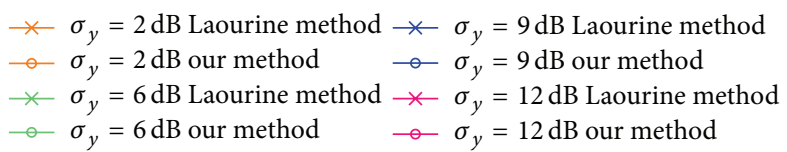

FIGURE 1: Ergodic capacity versus SNR for Laourine's method and our own method.

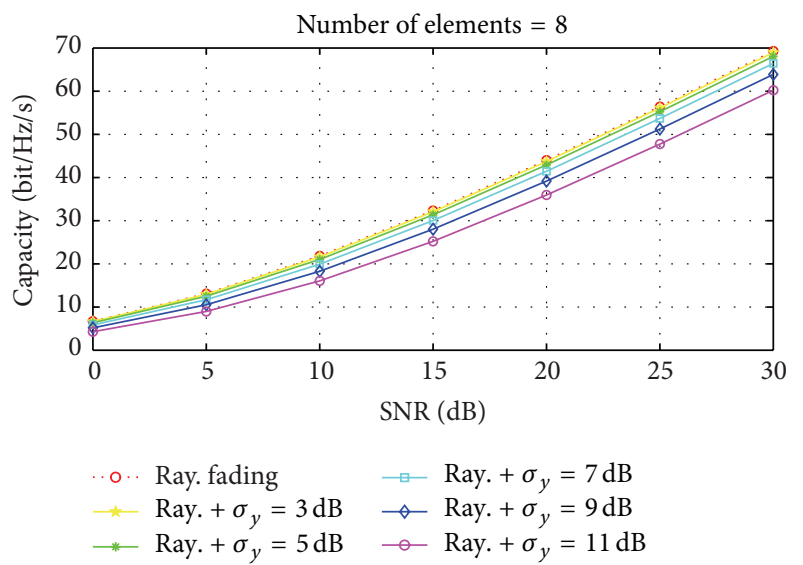

FIGURE 2: Capacity versus SNR for Rayleigh shadowing fading, with an $8 \times 8$ system.

In [13] Gauss-Hermite integration is used for presenting an approximated capacity expression. The work in [14] is centered on the analysis of the capacity with different adaptive schemes. In [15], it is proposed that shadowing can change the performance of MIMO systems and a preliminary analysis for D-MIMO systems with both fading effects is provided in [23]. However, in the works referred above, a study of the performance of D-MIMO capacity has not been found, and it is presented in this work.

Figure 2 shows the capacity versus SNR for Rayleigh fading plus shadowing in $8 \times 8$ systems. This plot includes the Rayleigh-only fading for comparison purposes. As it is expected when introducing the shadowing effect, a loss of capacity is observed. This loss increases when the $\sigma$ of shadowing is increased.

Figure 3 shows the capacity regarding the $\sigma$ of lognormal fading for different number of elements with SNR $=20 \mathrm{~dB}$. For the sake of comparison, the Rayleigh fading capacity is also included. In this figure, it is possible to observe that the shadowing behavior is very different with the variation of the number of elements, having a greater influence when

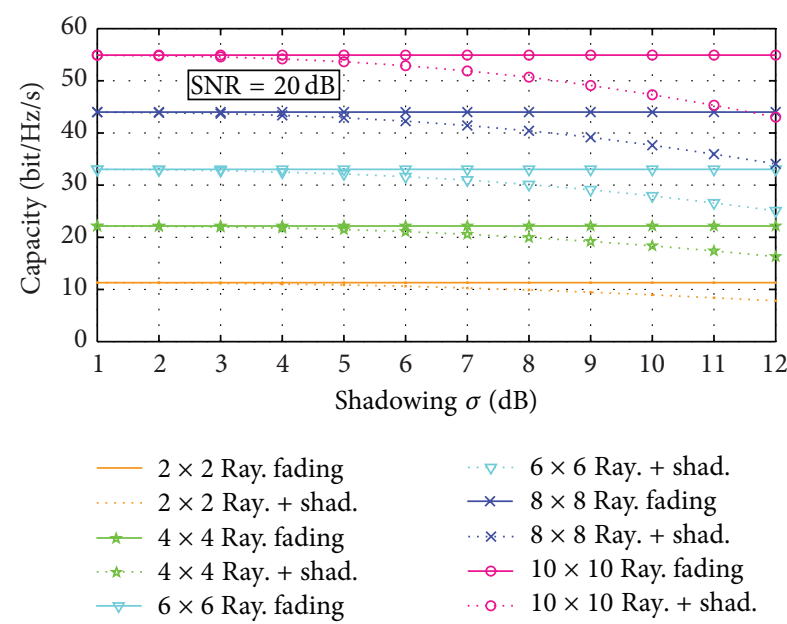

FIGURE 3: Capacity versus shadowing fading, with $\mathrm{SNR}=20 \mathrm{~dB}$.

the number of elements is higher. Figure 4 shows the decrease of capacity relative to Rayleigh-only fading regarding $\sigma$ shadowing for different number of elements with SNR = $10 \mathrm{~dB}$ (Figure 4(a)) and SNR $=25 \mathrm{~dB}$ (Figure 4(b)). This figure provides that the capacity decreases as the shadowing is increased. In this figure, it can be noticed that, for low SNR, the percentage of capacity loss is higher (for example, in the $2 \times 2$ MIMO system with SNR $=10 \mathrm{~dB}$ the capacity decrease is $40 \%$ while for SNR $=25 \mathrm{~dB}$ the capacity decrease is $26 \%$ ). These figures show the level in which the shadowing begins to affect the ergodic capacity of the D-MIMO channel, information which is quite useful for the system designer.

Figure 5 shows the capacity increase in relative percentage for Rayleigh fading versus the $\sigma$ of lognormal fading for different number of elements, with SNR $=25 \mathrm{~dB}$. Figure 6 shows the capacity increase regarding the number of elements and the $\sigma$ shadowing, on a 3D contour plot, for SNR = $10 \mathrm{~dB}$ (Figure 6(a)) and SNR $=25 \mathrm{~dB}$ (Figure 6(b)). From these figures, it can be concluded that for a high number of elements, the capacity loss is higher in absolute terms, but slightly lower in relative terms. Also, it is important to notice that the number of elements has more influence than the shadowing in the capacity value, as it is provided in Figure 6.

Finally in this section, different histograms are provided in Figure 7. In this figure, it is shown the probability distribution function of capacity in relation to the capacity of MIMO channel for a $10 \times 10$ system and SNR $=35 \mathrm{~dB}$. Also, in this figure it is plotted the mean capacity (with a dot) and the box-plot of capacity with the same color than the curve. It is observed that an increase in the shadowing level produces an increase in standard deviation of capacity; that is, the $\sigma$ increase produces the spread of the channel capacity.

\section{Results for Composite Channel (Rician + Shadowing)}

Rician fading occurs when one of the paths, typically a line of sight signal, is much stronger than the others. The $K$ factor is the results of dividing the higher path between the 


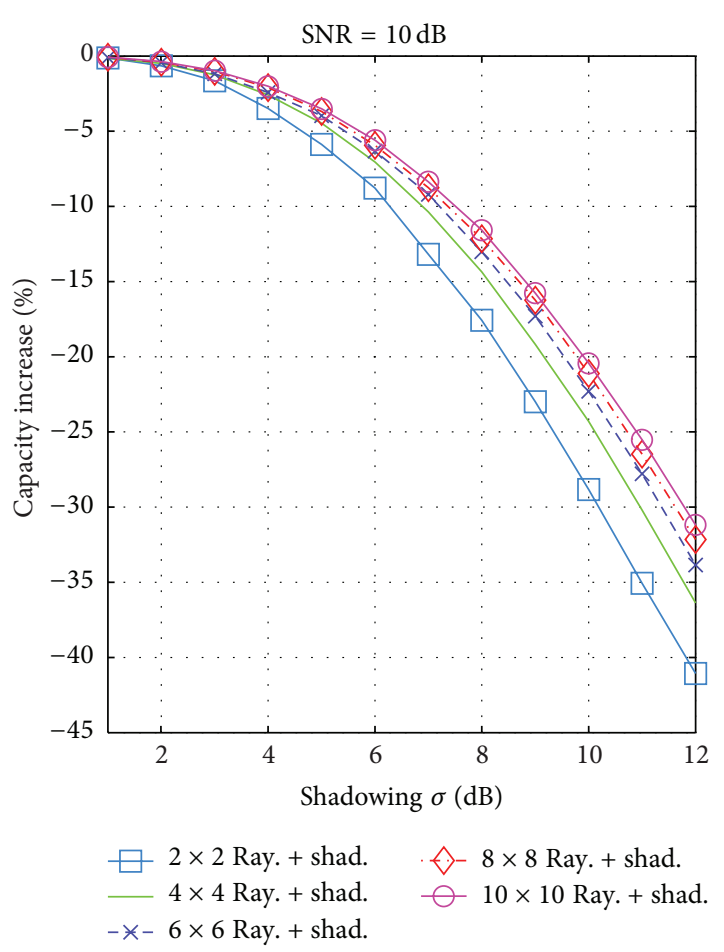

(a)

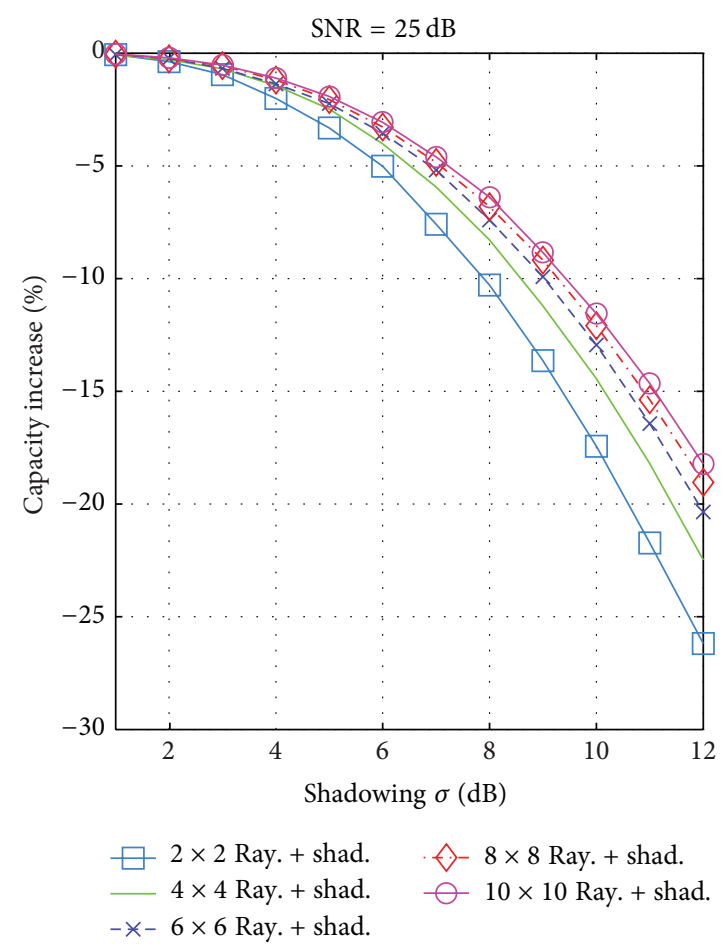

(b)

FIGURE 4: Capacity increase relative to Rayleigh-only fading versus sigma shadowing, (a) with SNR = $10 \mathrm{~dB},(\mathrm{~b})$ with $\mathrm{SNR}=25 \mathrm{~dB}$.

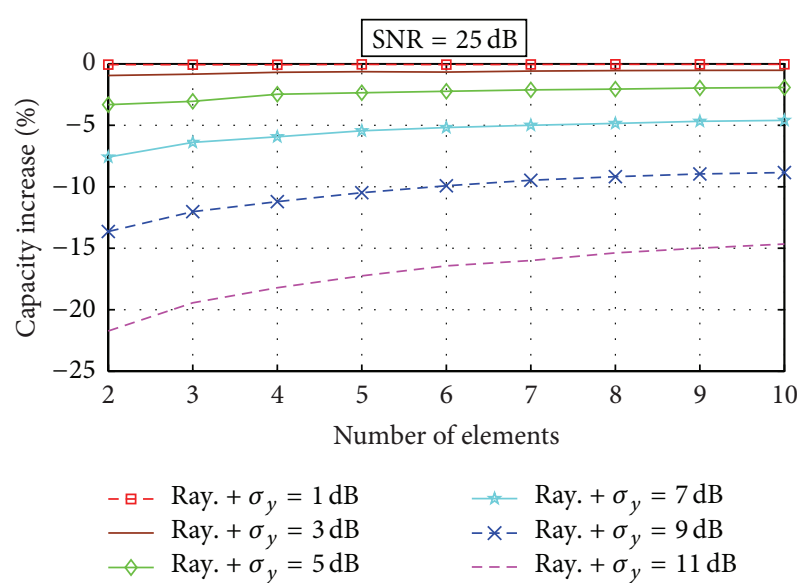

FIGURE 5: Capacity Increase versus number of elements shadowing $\sigma$ fading, with $\mathrm{SNR}=25 \mathrm{~dB}$.

sum of all others paths. The $K$-factor of more general Ricianfading environments changes as the distance of receiver to transmitter changes. The composite channel of Rician fading with shadowing is studied in [24, 25]. In [14], a semianalytical expression is presented for the probability density function and in [26]; new accurate formulas for obtaining symbol error outage are presented. However, in the literature, up to the authors' knowledge, no work about Shannon capacity for D-MIMO systems with a composite Rician-shadowed channel has been presented. In this section, in a similar way to the previous one, the Shannon capacity for the composite Rician plus shadowing channel is provided. Figure 8 shows the capacity regarding the SNR for this composite channel, with several $\sigma$ values and several $K$-factor values. It is remarkable that the Rician $K$-factor has more influence than the shadowing for this $4 \times 4$ example: it can be observed that the different lines are grouped regarding the $K$-factor (one group with diamonds $(K=-6 \mathrm{~dB})$ and the other with squares $(K=7 \mathrm{~dB}))$.

A statistical study of MIMO capacity is performed in systems under composite Rician fading. Figure 9 shows the probability density function of capacity considering the capacity for the $8 \times 8 \mathrm{MIMO}$ system, with $\mathrm{SNR}=30 \mathrm{~dB}$ and Rician $K$-factor $=-10 \mathrm{~dB}$ (Figure 9(a)), Rician $K$-factor $=$ $-0.5 \mathrm{~dB}$ (Figure 9(b)), Rician $K$-factor $=4.7 \mathrm{~dB}$ (Figure 9(c)), and Rician $K$-factor $=9 \mathrm{~dB}$ (Figure $9(\mathrm{~d})$ ). In this figure, it is also plotted the mean capacity (with a dot) and the box-plot of capacity with the same color than the curve. It is important to note that the increase in the shadowing severity produces an increase in the spread of the channel capacity. This agrees with previous results for $1 \times 1$ channels [27]. Moreover, it is observed that the mean value of the channel increases or decreases depending on the $K$-factor (see the different subfigures in Figure 9). All data of mean and standard deviation are depicted in Table 1 for the $8 \times 8 \mathrm{MIMO}$ system with $\mathrm{SNR}=30 \mathrm{~dB}$.

Figure 10 shows the probability density function of capacity regarding the capacity for the $8 \times 8$ MIMO system with $\mathrm{SNR}=30 \mathrm{~dB}$ and $\sigma=1 \mathrm{~dB}$ (Figure 10(a)), $\sigma=4 \mathrm{~dB}$ 


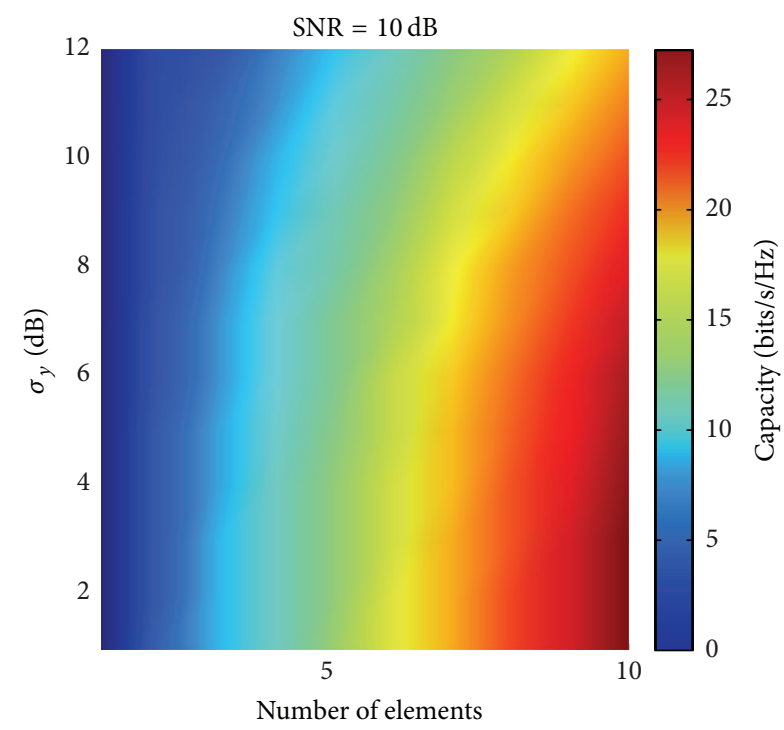

(a)

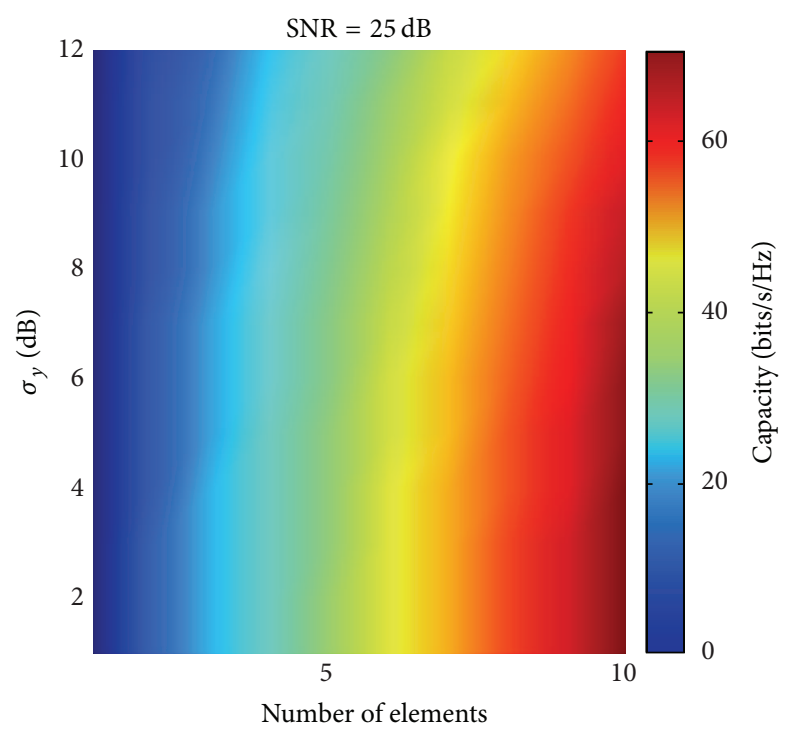

(b)

FIGURE 6: Capacity versus the number of elements versus $\sigma$ shadowing, on a 3D contour plot, (a) with SNR $=10 \mathrm{~dB}$ and (b), $\mathrm{SNR}=25 \mathrm{~dB}$.

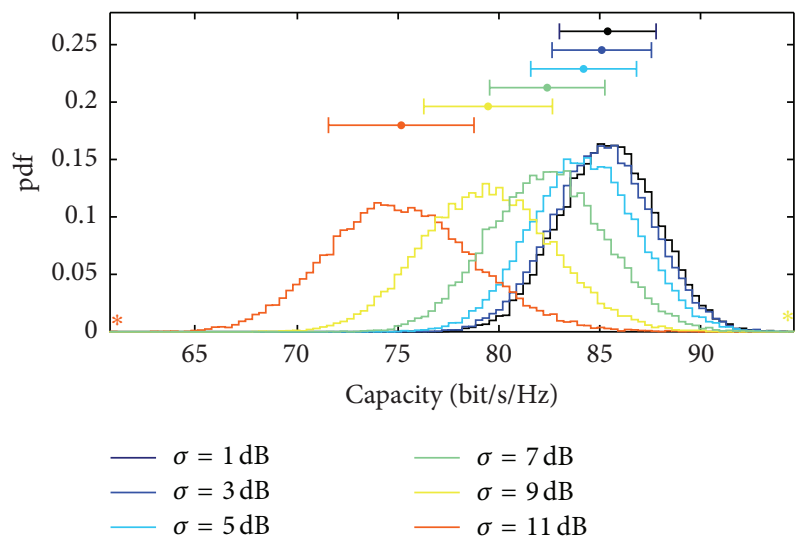

FIGURE 7: Histogram of different $10 \times 10$ MIMO systems with SNR $=$ $30 \mathrm{~dB}$.

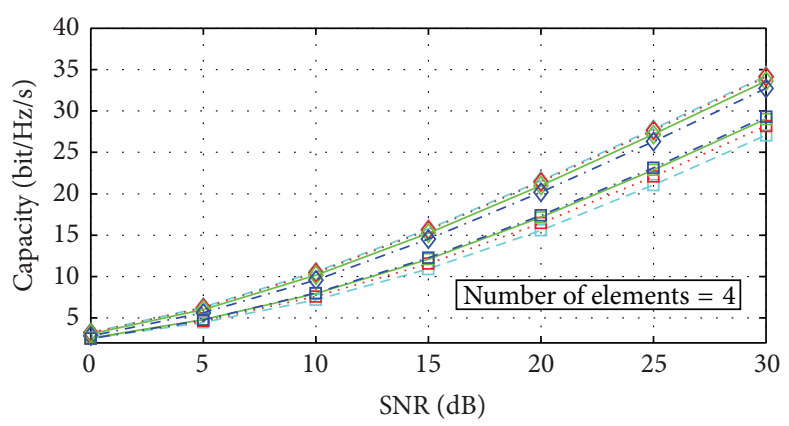

\footnotetext{
$-\diamond-K$-factor $=-6 \mathrm{~dB} \quad-\boxminus-K$-factor $=7 \mathrm{~dB}$

$\therefore \Delta . K$-factor $=-6 \mathrm{~dB}+\sigma_{y}=1 \mathrm{~dB} \cdots \boxminus \cdots K$-factor $=7 \mathrm{~dB}+\sigma_{y}=1 \mathrm{~dB}$

$\diamond-K$-factor $=-6 \mathrm{~dB}+\sigma_{y}=5 \mathrm{~dB}-\square-K$-factor $=7 \mathrm{~dB}+\sigma_{y}=5 \mathrm{~dB}$

$\diamond-K$-factor $=-6 \mathrm{~dB}+\sigma_{y}=9 \mathrm{~dB} \cdot--K$-factor $=7 \mathrm{~dB}+\sigma_{y}=9 \mathrm{~dB}$
}

FIGURE 8: Capacity versus SNR for Rician plus shadowing fading with several $\sigma$ and several $K$-factor values.
TABLE 1: Standard deviation and mean value of capacity for an $8 \times 8$ MIMO system with SNR $=30 \mathrm{~dB}$ in composite fading.

\begin{tabular}{lcccccccc}
\hline & \multicolumn{2}{c}{$K=-10 \mathrm{~dB}$} & \multicolumn{2}{c}{$K=-0.5 \mathrm{~dB}$} & \multicolumn{2}{c}{$K=4.7 \mathrm{~dB}$} & \multicolumn{2}{c}{$K=9 \mathrm{~dB}$} \\
& Mean & Std. & Mean & Std. & Mean & Std. & Mean & Std. \\
\hline $1 \mathrm{~dB}$ & 68.5 & 2.4 & 61.7 & 2.2 & 51.4 & 1.9 & 46.0 & 1.7 \\
$3 \mathrm{~dB}$ & 68.3 & 2.4 & 62.0 & 2.3 & 53.4 & 2.0 & 49.6 & 2.0 \\
$5 \mathrm{~dB}$ & 67.5 & 2.6 & 62.0 & 2.5 & 55.4 & 2.4 & 53.1 & 2.3 \\
$7 \mathrm{~dB}$ & 65.9 & 2.8 & 61.3 & 2.7 & 56.4 & 2.7 & 55.0 & 2.7 \\
$9 \mathrm{~dB}$ & 63.4 & 3.1 & 59.7 & 3.1 & 56.2 & 3.1 & 55.2 & 3.1 \\
$11 \mathrm{~dB}$ & 59.9 & 3.5 & 57.0 & 3.5 & 54.6 & 3.5 & 54.0 & 3.5 \\
\hline
\end{tabular}

(Figure 10(b)) $\sigma=7 \mathrm{~dB}$ (Figure 10(c)), and $\sigma=10 \mathrm{~dB}$ (Figure 10(d)). In this figure, it is observed that the different curves in the same figure have similar standard deviation; that is, the channel with the same shadowing has similar spread of the channel capacity. In addition, it is possible to note that the increase of Rician $K$-factor produces a decrease of mean capacity (the distribution curve moves towards the left) and a slight reduction of the spread of the channel capacity. This reduction when the $K$-factor is increased agrees with the reported results in [28]. This effect decreases when the $\sigma$ is increased (for example the standard deviation change when $\sigma=1 \mathrm{~dB}$ from 2.4 with $K$-factor $=-10 \mathrm{~dB}$ to 1.7 with $K=10 \mathrm{~dB}$, although when $\sigma=11 \mathrm{~dB}$ the standard deviation is constant for all Rician $K$-factor values). That is, when the $K$-factor is increased the spread of the channel capacity is decreased. However, this effect is neglected when the shadowing is present with a high $\sigma$ value. Thus, the effect of Rician fading can be compensated with the effect of Shadowing. This fact takes some advantage of the systems that work under Rician composite fading from the systems that work only under Rician-only fading, that is, D-MIMO systems from MIMO systems. Figure 11 shows the capacity 


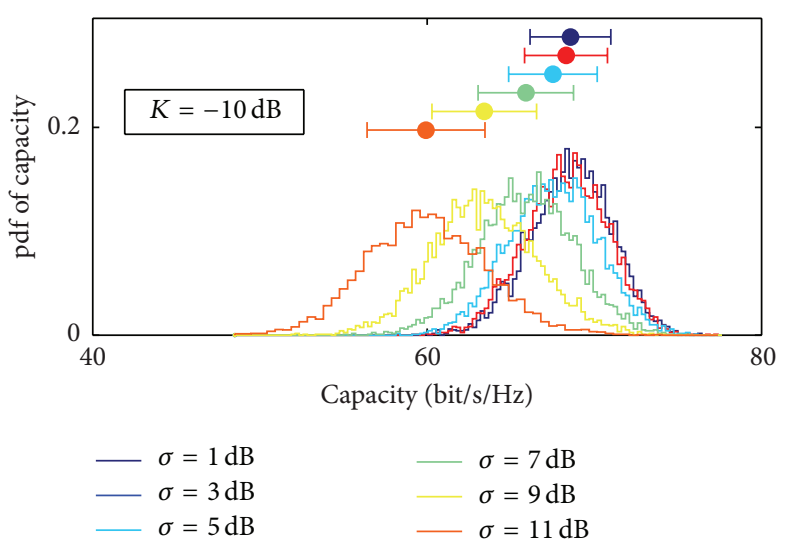

(a)

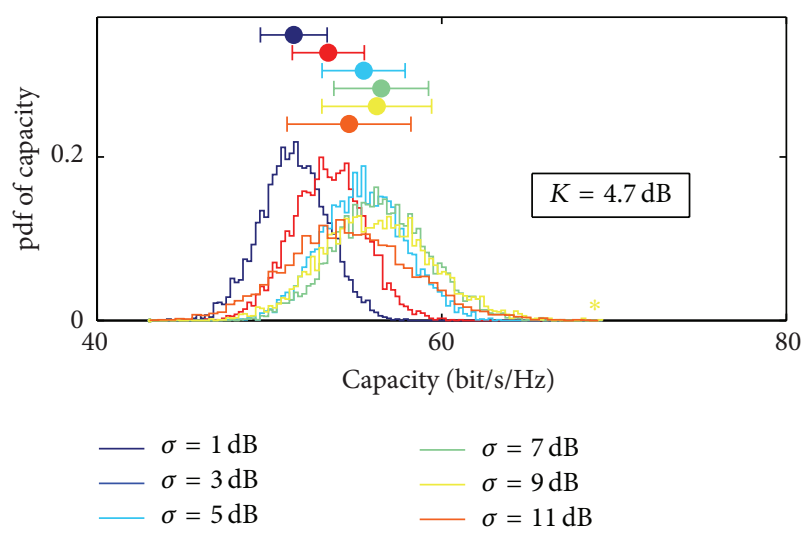

(c)

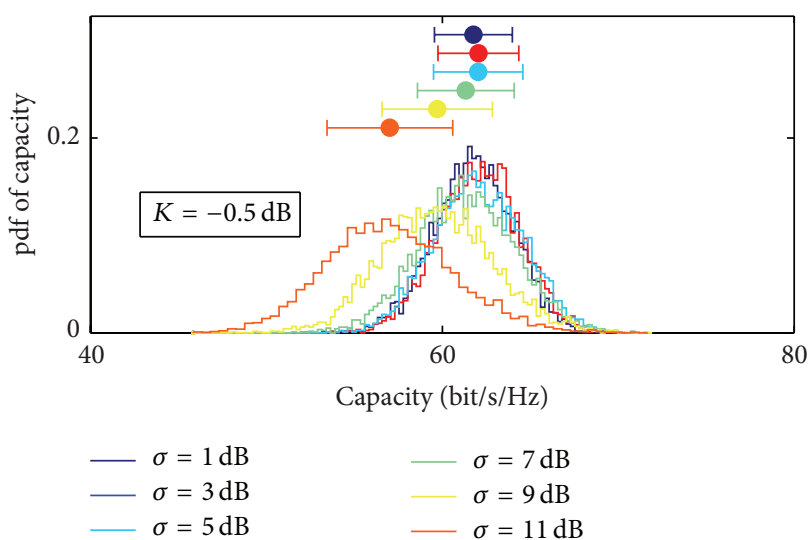

(b)

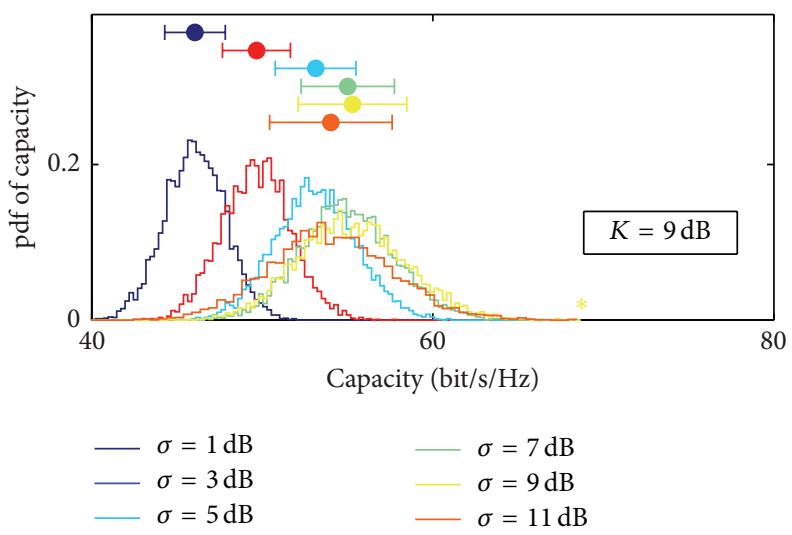

(d)

FIGURE 9: Probability density function of capacity versus capacity for $8 \times 8$ MIMO system, (a) with SNR $=30 \mathrm{~dB}$ and Rician $K$-factor $=$ $-10 \mathrm{~dB}$, (b) Rician $K$-factor $=-0.5 \mathrm{~dB}$, (c) Rician $K$-factor $=4.7 \mathrm{~dB}$, and (d) Rician $K$-factor $=9 \mathrm{~dB}$.

versus $K$-factor, with the shadowing as a parameter, for a $2 \times 2$ (Figure 11(a)), a $3 \times 3$ (Figure 11(b)), a $4 \times 4$ (Figure 11(c)), and a $5 \times 5$ (Figure 11(d)) system. In this figure, it is possible to observe that the Rician-only fading (red line) suffers more degradation with the increase of $K$-factor than the Rician composite fading (the rest of lines). In these figures, it is possible to observe that, for a low $K$-factor (similar to the Rayleigh case), the shadowing decreases the capacity in all the cases. In this figure, it can be also noticed that the lowest capacity degradation, when the $K$-factor is increased, is produced for the higher $\sigma$ shadowing values. This is due to the environment that has been already degraded with the shadowing and it is difficult to degrade more the signal.

It is remarkable that the shadowing has more influence than the Rician $K$-factor for $2 \times 2$ systems. However, when the number of elements is increased this situation changes: for a $3 \times 3$ system the influence of both phenomena is similar and for a $4 \times 4$ and $5 \times 5$ system the Rician $K$-factor has more influence than the shadowing. This phenomenon, observed in this research for the first time, is a key result and one of the main contributions of this paper. That is, for a high $K$-factor, the shadowing can compensate the lack of scattering that is produced in Rician-only fading.
In other words, when the $K$-factor is high, the line-ofsigh (LOS) component is predominant; however, when the shadowing is introduced the LOS is lost, that is, the effect of shadowing compensates the effect of Rician fading. Thus, when the shadowing is introduced some paths between the transmitters and receivers are blocked and some new paths are added (not always), producing a new scenario. This fact is reflected in the increase of the capacity spread (the standard deviation of capacity increases its value), and an increase in the mean capacity is produced. As a result, it is reported for the first time that shadowing can increase the capacity of $\mathrm{D}$ MIMO systems.

The following figures (Figures 12 and 13) show up to what extent the shadowing level begins to increase the ergodic capacity of the D-MIMO channel, information which is of interest for the system designer. Figure 12 shows the capacity increase relative to Rician-only channel versus the number of elements, with shadowing as a parameter, for different $K$-factor values: $K=-7 \mathrm{~dB}$ (Figure $12(\mathrm{a})$ ), $K=2 \mathrm{~dB}$ (Figure 12(b)), $K=6 \mathrm{~dB}$ (Figure 12(c)), and $K=10 \mathrm{~dB}$ (Figure 12(d)). It is remarkable that in Figure 12(a) with $K=$ $-7 \mathrm{~dB}$, there is not positive capacity increase. However, when the $K$-factor is increased correlatively in Figures 12(b), 12(c), 

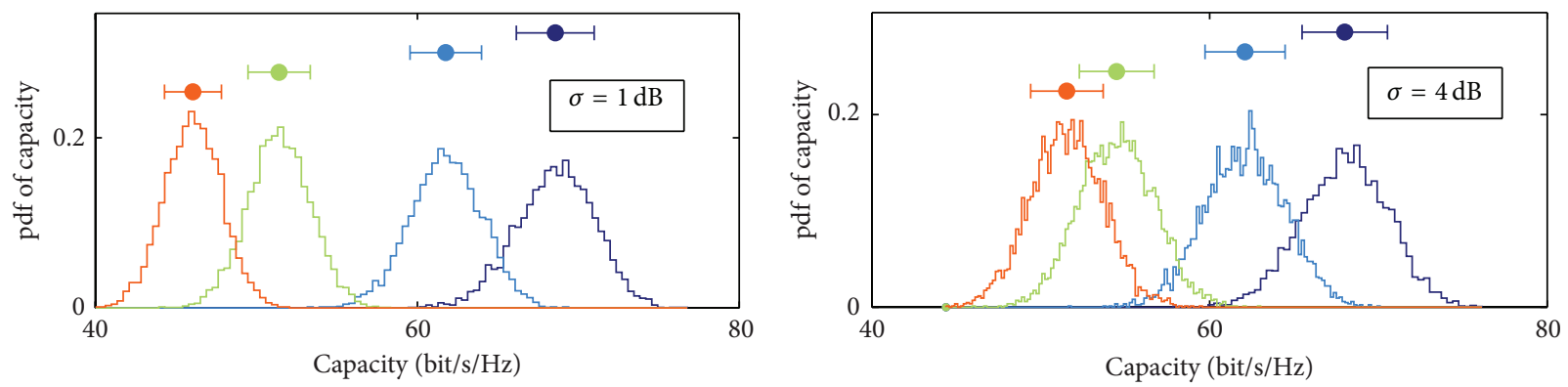

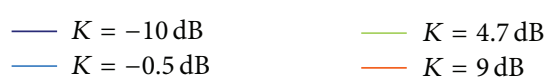

(a)

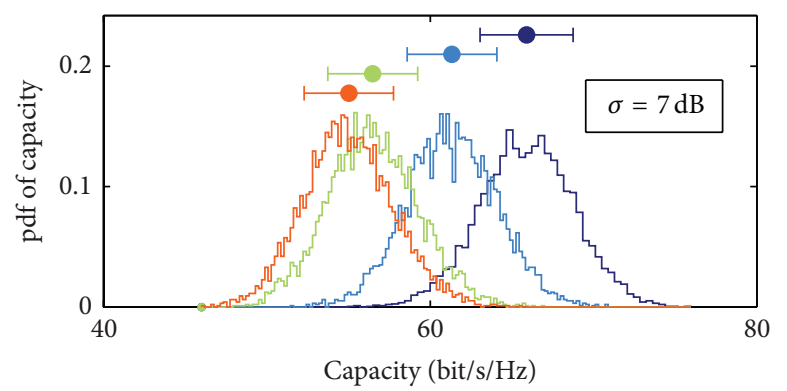

$\begin{aligned} K & =-10 \mathrm{~dB} & -K & =4.7 \mathrm{~dB} \\ K & =-0.5 \mathrm{~dB} & -K & =9 \mathrm{~dB}\end{aligned}$

(c)

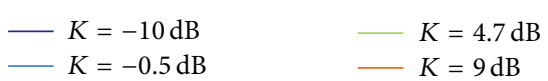

(b)

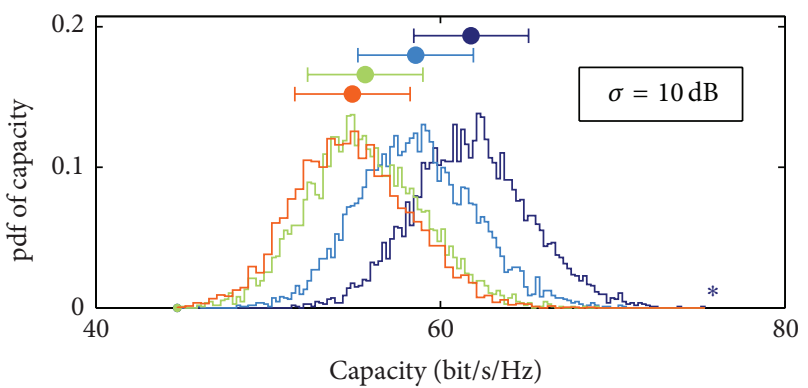

$\begin{aligned} K & =-10 \mathrm{~dB} \\ K & =-0.5 \mathrm{~dB}\end{aligned}$

$K=4.7 \mathrm{~dB}$

$K=9 \mathrm{~dB}$

(d)

FIGURe 10: Probability density function of capacity versus capacity for the $8 \times 8$ MIMO system, (a) with $\mathrm{SNR}=30 \mathrm{~dB}$ and $\sigma=1 \mathrm{~dB}$, (b) $\sigma=4 \mathrm{~dB},(\mathrm{c}) \sigma=7 \mathrm{~dB}$, and (d) $\sigma=10 \mathrm{~dB}$.

and $12(\mathrm{~d})$, the increment of capacity is positive for some values and higher when the $K$-factor is higher. Also, it is possible to notice that the capacity increase is positive as a general rule when both the $K$-factor and the number of elements are high. Thus, in some cases, the capacity of the Rician-shadowed channel exceeds the one of the Rician-only channel. That is, the D-MIMO system with Rician fading and distributed antennas that experiences log-normal shadowing can exceed the capacity of traditional MIMO systems with Rician-only channel.

Figure 13 shows the capacity increase (\%) regarding the $K$-factor and shadowing, in a 3D contour plot, with SNR = $5 \mathrm{~dB}$ and number of elements equal to 2 (Figure 13(a)), with $\mathrm{SNR}=5 \mathrm{~dB}$ and 7 elements (Figure 13(b)), with SNR $=20 \mathrm{~dB}$ and 2 elements (Figure 13(c)), and with SNR $=20 \mathrm{~dB}$ and 7 elements (Figure 13(d)). In this figure, it is noticed that the capacity increase depends on the number of elements and the SNR value because the four different plots have great difference. In Figures 13(a) and 13(c) with low number of elements, the capacity increase is almost constant with the $K$-factor and only varies with the shadowing. However, in Figures 13(b) and 13(d), when the number of elements is 7, the maximum increment (red zone) is concentrated for a high $K$-factor. It is also observed that, with small number of elements, there is generally a decrease in capacity relative to the Rician-only channel. With a large number of elements there can be a substantial increase. As a conclusion, D-MIMO systems with log-normal shadowing and Rician fading have a clear advantage over MIMO systems that experience Rician fading only in specific conditions (mainly for high number of elements and high $K$-factor).

\section{Correlated and Experimental Results}

6.1. Correlated Results. Typically, the $H_{\text {micro }}$ is calculated with (2), forming an uncorrelated $H_{\text {micro }}$ matrix. However, in this section, it is tried to test a correlated MIMO distributed system. Thus, it is necessary to generate one correlated $H_{\text {micro }}$ matrix. The procedure (method) to generate the correlated $H_{\text {micro }}$ matrix is as follows.

(1) $P$ realizations of vector $L$ with dimensions $1 \times T$ are generated. The number $P$ varies and is higher than 10 :

$$
\begin{aligned}
L_{1} & =f_{\mathrm{Nor}}(1, T)+j * f_{\mathrm{Nor}}(1, T) \\
L_{2} & =f_{\mathrm{Nor}}(1, T)+j * f_{\mathrm{Nor}}(1, T) \\
& \vdots \\
L_{P} & =f_{\mathrm{Nor}}(1, T)+j * f_{\mathrm{Nor}}(1, T) .
\end{aligned}
$$

(2) A random vector $W$, between 0 and 1 and with dimensions $1 \times P$, that represents different weights, 


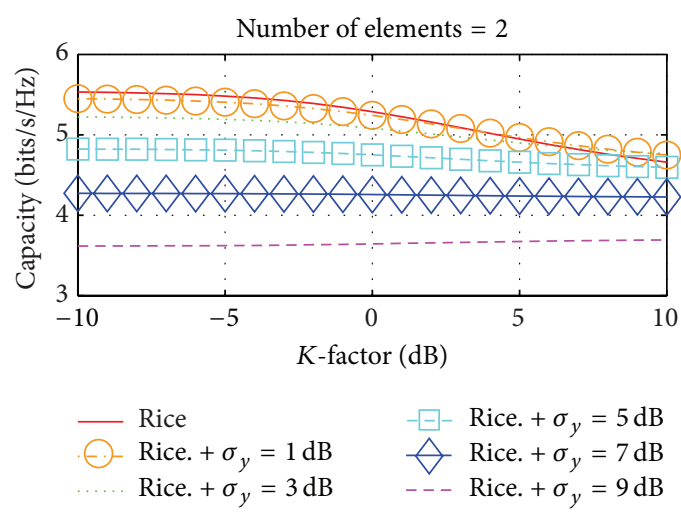

(a)

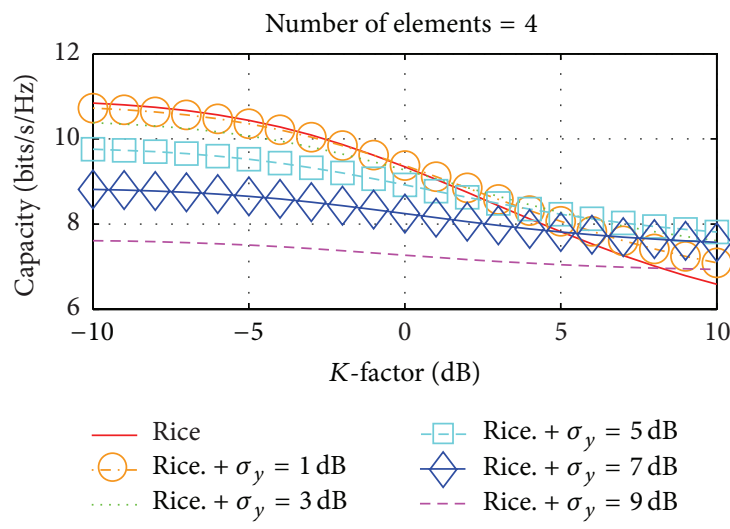

(c)

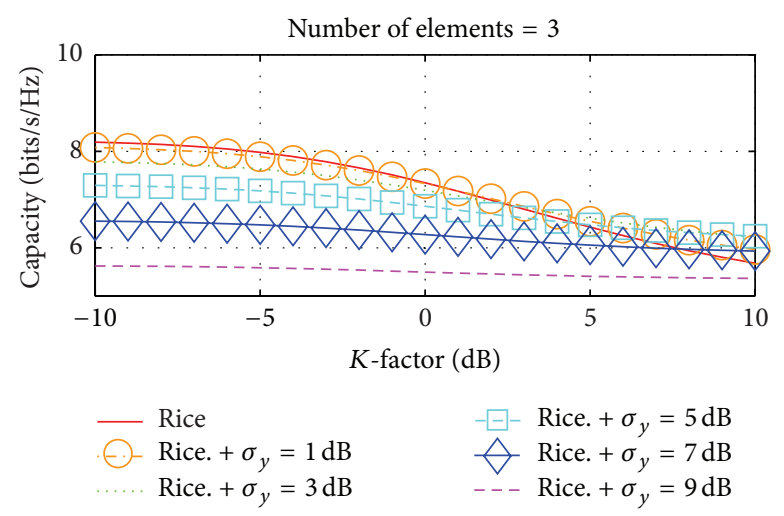

(b)

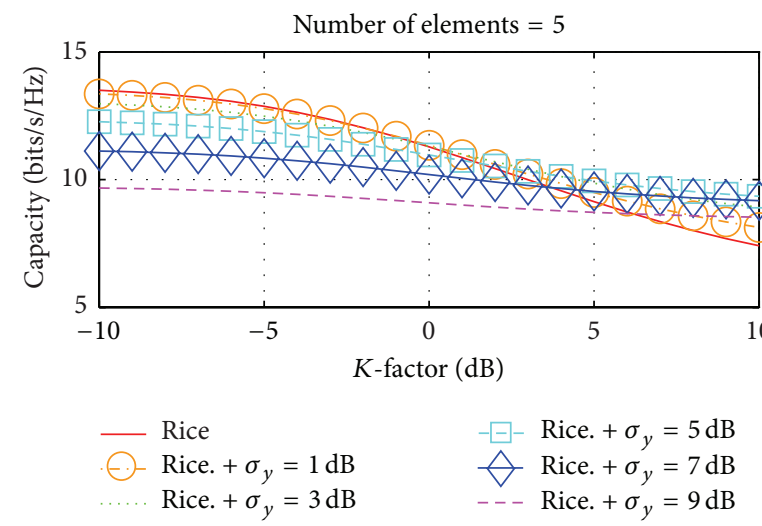

(d)

FIgURE 11: Capacity versus $K$-factor with shadowing as parameter, (a) for $2 \times 2$, (b) $3 \times 3$, (c) $4 \times 4$, and (d) $5 \times 5$ systems.

will be used to combine different $L$ vectors in a fixed way:

$$
W=f_{\text {Nor }}(1, P) \text {. }
$$

(3) A fixed vector $B$, with dimensions $(1 \times T)$, combination of $L$ vectors, is calculated. This term is calculated in order to introduce high correlation:

$B=L_{1} * W(1)+L_{2} * W(2)+\cdots+L_{P} * W(P)$.

(4) A set of $R$ vectors " $W W$," which are random vectors between 0 and 1 with dimensions $1 \times P$, is defined. These vectors represent different weights, which will be used to be combined with the different $L$ vectors:

$$
\begin{aligned}
W W_{1} & =f_{\text {Nor }}(1, P) \\
W W_{2} & =f_{\text {Nor }}(1, P) \\
& \vdots \\
W W_{R} & =f_{\text {Nor }}(1, P) .
\end{aligned}
$$

(5) A set of $R$ vectors " $B B$," one for each reception antenna, is calculated in order to introduce low correlation; these matrices are a linear combination of
$L$ matrices weighted with the different $W W$ vectors. That is, for 1 to $R$, it is done:

$$
\begin{aligned}
B B_{1} & =L_{1} * W W_{1}(1)+L_{2} * W W_{1}(2)+\cdots+L_{P} * W W_{1}(P) \\
B B_{2} & =L_{1} * W W_{2}(1)+L_{2} * W W_{2}(2)+\cdots+L_{P} * W W_{2}(P) \\
& \vdots \\
B B_{R} & =L_{1} * W W_{R}(1)+L_{2} * W W_{R}(2)+\cdots+L_{P} * W W_{R}(P) .
\end{aligned}
$$

(6) A set of $R$ random vectors " $A A$," one for each reception antenna, is calculated in order to randomize. That is, for 1 to $R$ it is done:

$$
\begin{aligned}
A A_{1} & =f_{\mathrm{Nor}}(1, T)+j * f_{\mathrm{Nor}}(1, T) \\
A A_{2} & =f_{\mathrm{Nor}}(1, T)+j * f_{\mathrm{Nor}}(1, T) \\
& \vdots \\
A A_{R} & =f_{\mathrm{Nor}}(1, T)+j * f_{\mathrm{Nor}}(1, T) .
\end{aligned}
$$

(7) Finally, to calculate each $H H$ vector, the previous calculated vectors $(B, B B$, and $A A)$ are combined in 


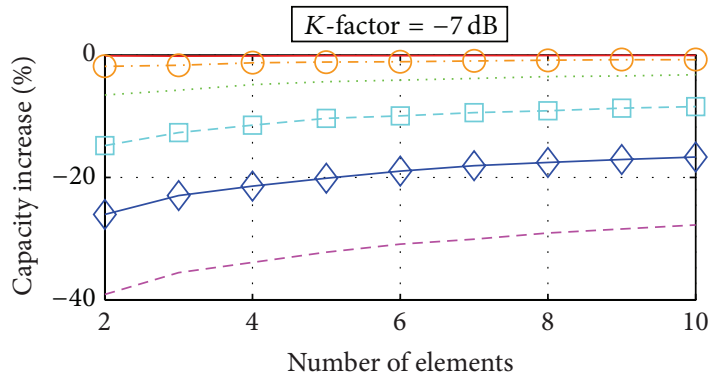

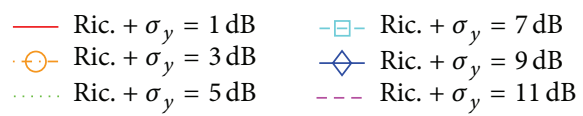

(a)

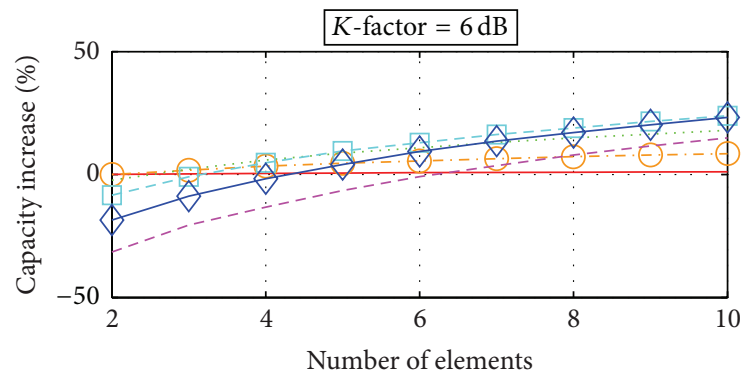

$\begin{array}{ll}\text { Ric. }+\sigma_{y}=1 \mathrm{~dB} & - \text { Ric. }+\sigma_{y}=7 \mathrm{~dB} \\ \text { Ric. }+\sigma_{y}=3 \mathrm{~dB} & - \text { Ric. }+\sigma_{y}=9 \mathrm{~dB} \\ \text { Ric. }+\sigma_{y}=5 \mathrm{~dB} & --- \text { Ric. }+\sigma_{y}=11 \mathrm{~dB}\end{array}$

(c)

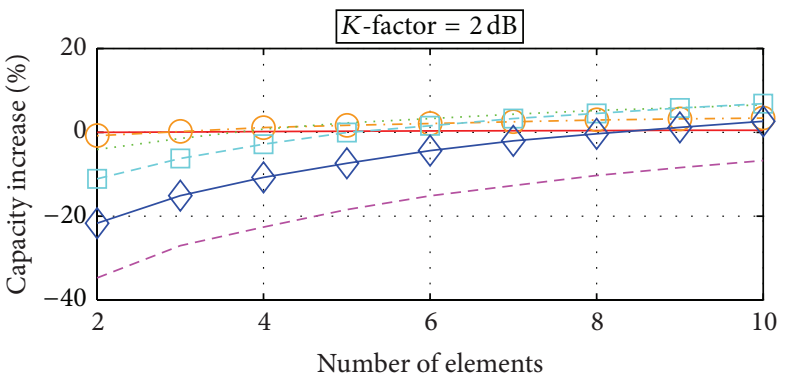

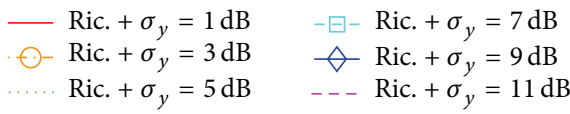

(b)

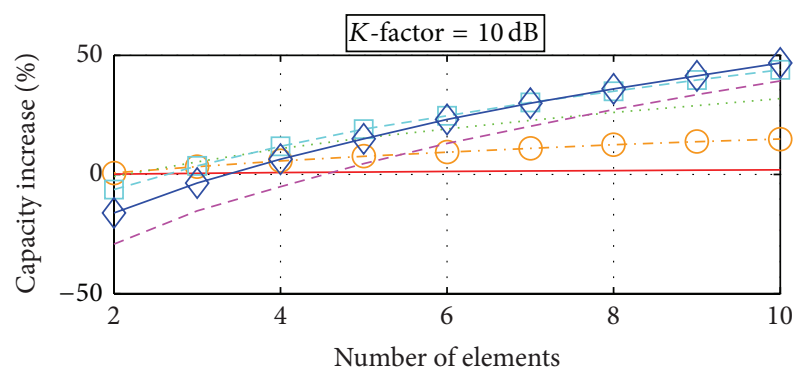

(d)

FIGURE 12: Capacity increase versus the number of elements, with shadowing as parameter and for different $K$-factor, (a) $K=-7 \mathrm{~dB}$, (b) $K=2 \mathrm{~dB}$, (c) $K=6 \mathrm{~dB}$, and (d) $K=10 \mathrm{~dB}$.

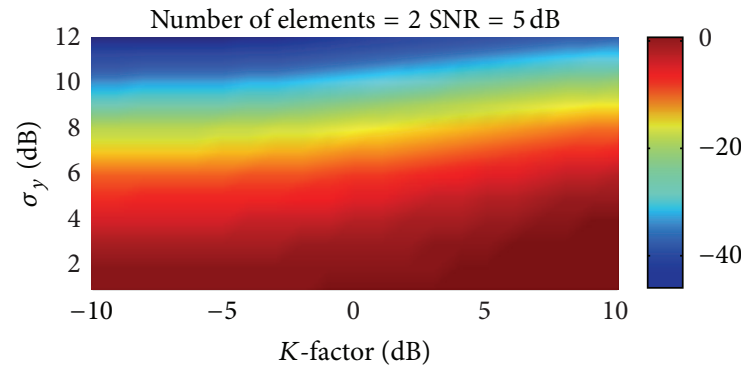

(a)

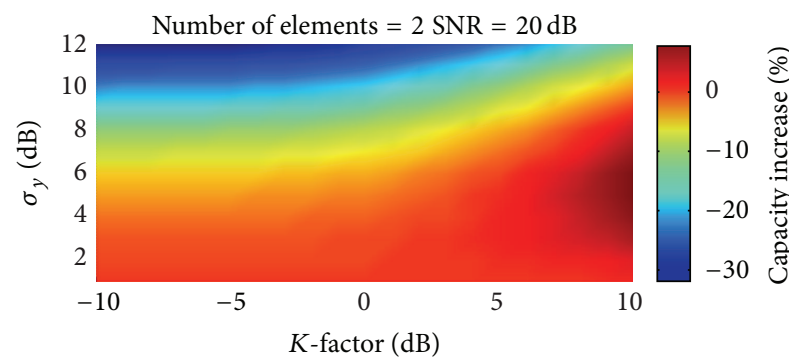

(c)

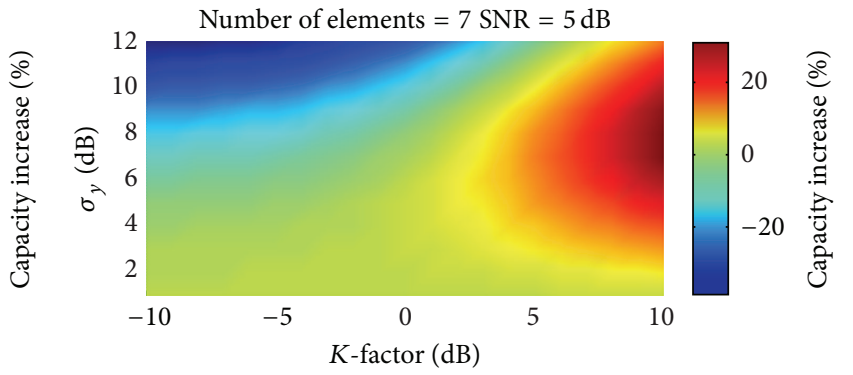

(b)

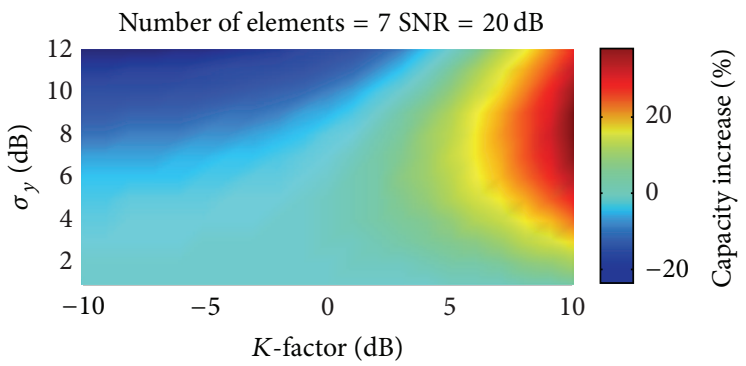

(d)

FIGURE 13: Capacity increase (\%) versus the $K$-factor and versus shadowing, (a) with $\mathrm{SNR}=5 \mathrm{~dB}$ and number of elements equal to 2 , (b) $\mathrm{SNR}=5 \mathrm{~dB}$ and 7 elements, (c) SNR $=20 \mathrm{~dB}$ and 2 elements, and (d) $\mathrm{SNR}=20 \mathrm{~dB}$ and 7 elements. 


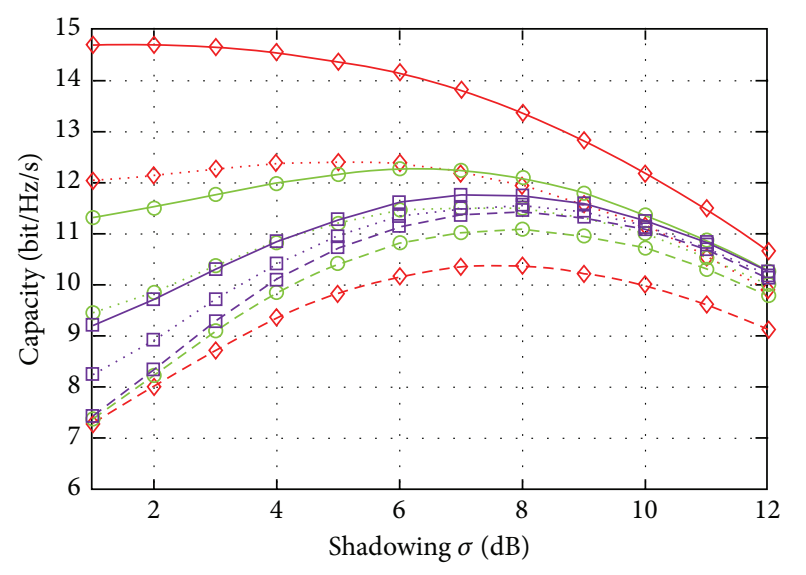

$-\diamond-$ Rayleigh mean corr. $=0.93$
$\diamond$. Rayleigh mean corr. $=0.62$
$\diamond-$ Rayleigh mean corr. $=0.05$
$-\ominus-$ Mean corr. $=0.93 \mathrm{~K}$-factor $=3 \mathrm{~dB}$
$\therefore$. Mean corr. $=0.62 \mathrm{~K}$-factor $=3 \mathrm{~dB}$
- Mean corr. $=0.05 \mathrm{~K}$-factor $=3 \mathrm{~dB}$
$-\boxminus-$ Mean corr. $=0.93 \mathrm{~K}$-factor $=9 \mathrm{~dB}$
$\cdots \square$. Mean corr. $=0.62 \mathrm{~K}$-factor $=9 \mathrm{~dB}$
$\square-$ Mean corr. $=0.05 \mathrm{~K}$-factor $=9 \mathrm{~dB}$

FIGURE 14: Distributed MIMO capacity versus shadowing level for correlated system with composite fading, Rayleigh and Rician (with different $K$-factor).

a weighed way. That is, $H H$ vectors are composed by a fixed term " $B$," a high correlated term " $B B$," and a random term " $A A$." Thus, modifying the scalar values $(a, b$, and $c)$, it is possible to obtain any correlation for $H$ matrix. That is, for 1 to $R$ it is done:

$$
\begin{aligned}
H H_{1} & =a * B+b * B B_{1}+c * A A_{1} \\
H H_{2} & =a * B+b * B B_{2}+c * A A_{2} \\
& \vdots \\
H H_{R} & =a * B+b * B B_{R}+c * A A_{R} .
\end{aligned}
$$

(8) The $H$ matrix is generated concatenating the different $H H$ matrix from 1 to $R$ :

$$
H_{\text {micro }}=\left[H H_{1}, H H_{2} \cdots H H_{R}\right] .
$$

(9) The $H_{\text {micro }}$ matrix is normalized with Frobenius norm in order to obtain one $H_{\text {micro }}$ matrix with zero mean.

Thus, it is possible to generate the $H_{\text {micro }}$ channel matrix with Rayleigh distribution with any number of elements $(R$ and $T)$ and any level of correlation. From $H_{\text {micro }}$, it is possible to generate any $H_{\text {micro }}$ channel matrix with Rician distribution, with the same procedure than the one developed in [29] or provided in the previous section. Thus, with the model developed in Section 2 it is possible to compute any $H$ matrix with correlated composite fading (Rayleigh/Rician plus shadowing).
Figure 14 shows a $4 \times 4$ system with different correlation and different composite fading (Rayleigh and Rician with different $K$-factor). In order to simplify, the mean value of all antenna pair is the value taken. In this figure, it is possible to observe than the correlation affects more to Rayleigh composite fading than to Rician fading. Also, it is possible to see that, in correlated systems, it is possible to increase capacity for some shadowing values even for Rayleigh fading. Further results for other propagation models may be found in [30].

6.2. Experimental Results. In this subsection, the model proposed above to is applied measured data in reverberation chamber (RC). Therefore, the composite fading for Rayleigh/Rician plus shadowing is obtained for the data measured in RC. With this model it is possible to emulate composite fading in the RC, in a similar way to [16], where a postprocess technique is proposed to emulate the Rician channel on the RC. In this case, the $H_{\text {micro }}$ in (2) is in fact a real matrix measured in RC. It is also very interesting that this type of fading is also proposed for massive MIMO $[29,31]$ and these experimental results open the door to emulate massive MIMO on RC.

In order to obtain experimental results, different nonisotropic scenarios made in [32, 33] are evaluated. Three different systems are evaluated; for system A, an empty RC is used, providing the typical isotropic scattering (Scenario A of [32]). For system B, one piece of absorber is used, consisting of 24 cones, placed on the right hand side wall of the chamber (Scenario B of [32]). Two $30 \mathrm{~cm}$ high hollow cylinders of $5 \mathrm{~cm}$ radius, made of Teflon (PTFE) and filled with lossy CENELEC head simulating liquid (HSL) at the frequency of interest, are introduced in the empty chamber to provide the setup of system $\mathrm{C}$ (Scenario D of [32]). The antenna spacing for the array is $0.25 \mathrm{~d} / \lambda$ in all scenarios, for more information on the measurement setup, the reader is referred to [32]. Figure 15 shows the distributed MIMO capacity for $3 \times 3$ systems $\mathrm{A}, \mathrm{B}$, and $\mathrm{C}$ with composite fading, that is, Rayleigh and Rician (with different $K$-factor) plus different shadowing level. In this figure, the same behavior for measured systems is observed, compared to the simulated results from previous sections. That is, the shadowing can increase the capacity in some conditions, as it is reported previously.

\section{Conclusions}

In this paper, the capacity behavior of a D-MIMO system for a composite Rayleigh shadowing channel has been studied. Experimentation for different shadowing intensity levels is provided, showing a wide variety of results of these systems, depending on the number of elements and the SNR. These results show up to what extent shadowing level begins to affect the ergodic capacity of the D-MIMO channel, information which is of interest for the system designer.

In addition, for the first time (in similar way to Rayleigh fading), results of ergodic capacity of D-MIMO system for a composite Rician shadowing fading are provided for different shadowing intensity levels showing a wide variety of results 


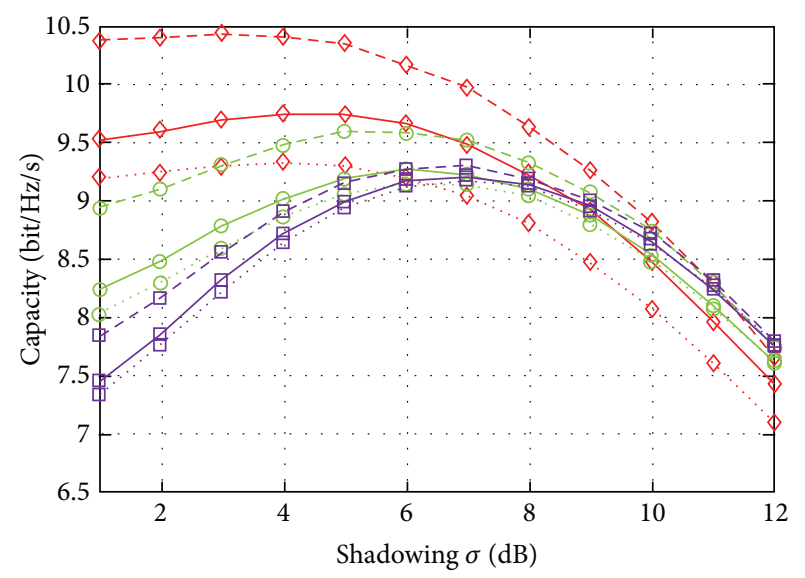

$$
\begin{aligned}
& -\diamond-\text { System A Ray. } \\
& \text { •. System B Ray. } \\
& \checkmark \text { System C Ray. } \\
& -\ominus-\text { System A } K \text {-factor }=3.0 \mathrm{~dB} \\
& \text {. . System B K-factor }=3.0 \mathrm{~dB} \\
& \multimap \text { System C } K \text {-factor }=3.0 \mathrm{~dB} \\
& \text {-曰- System A K-factor }=9.0 \mathrm{~dB} \\
& \text { 曰. System B } K \text {-factor }=9.0 \mathrm{~dB} \\
& \square \text { System C } K \text {-factor }=9.0 \mathrm{~dB}
\end{aligned}
$$

FIGURE 15: Distributed MIMO capacity for system A, B, and C versus shadowing level for composite fading, Rayleigh and Rician (with different $K$-factor).

of these systems, depending on the number of elements and the SNR. In addition, a statistic study of the channel capacity has been performed, which lets us identify how the shadowing can compensate the effect of Rician fading. This makes us conclude that when the $K$-factor is high, the lineof-sigh (LOS) component is predominant. However, when the shadowing is introduced, the LOS is lost and the effect of shadowing compensates the effect of Rician fading. This fact takes some advantage of the systems that work under Rician composite fading from the systems that work under Ricianonly fading, that is, D-MIMO systems from MIMO systems. Thus, it is identified that the D-MIMO systems can benefit from shadowing in Rician channels. This advantage has not been reported previously.

The D-MIMO system with Rician fading and distributed antennas that experience log-normal shadowing can exceed the capacity of traditional MIMO systems with Ricianonly channel. Thus, in a similar way, as it happened with MIMO systems, considering multipath propagation (initially considered to be a restriction but now turned to be the solution to reach higher capacities), the cooperative networks can take advantage of shadowing, which has been typically considered as a restriction in order to reach higher capacities. In addition, correlated and experimental results have been shown. More studies on the correlated and experimental results will be done in future work.

\section{Conflict of Interests}

The authors declare that there is no conflict of interests regarding the publication of this paper.

\section{Acknowledgments}

This work has been supported by "Gobierno de Extremadura" with project number IB13113, PYR-2014 GENIL project (PYR-2014-CEB09-0010/MICINN), and CEIbioTIC project (mP_TIC_11).

\section{References}

[1] G. L. Turin, F. D. Clapp, T. L. Johnston, S. B. Fine, and D. Lavry, "A statistical model of urban multipath propagation," IEEE Transactions on Vehicular Technology, vol. 21, no. 1, pp. 1-9, 1972.

[2] F. Hansen and F. I. Meno, "Mobile fading Rayleigh and lognormal superimposed," IEEE Transactions on Vehicular Technology, vol. 26, no. 4, pp. 332-335, 1977.

[3] F. F. Digham and M.-S. Alouini, "Outage probability of selection diversity in an exponentially correlated lognormal environment," in Proccedings of the 5th Nordic Signal Processing Symposium, October 2002.

[4] S. Cui, A. J. Goldsmith, and A. Bahai, "Energy-efficiency of MIMO and cooperative MIMO techniques in sensor networks," IEEE Journal on Selected Areas in Communications, vol. 22, no. 6, pp. 1089-1098, 2004.

[5] A. Ozgur, O. Leveque, and D. N. Tse, "Hierarchical cooperation achieves optimal capacity scaling in ad hoc networks," IEEE Transactions on Information Theory, vol. 53, no. 10, pp. 35493572, 2007.

[6] M. V. Clark, T. M. Willis III, L. J. Greenstein, A. J. Rustako, V. Erceg, and R. S. Roman, "Distributed versus centralized antenna arrays in broadband wireless networks," in Proceedings of the IEEE Vehicular Technology Conference (VTC '01), pp. 3337, May 2001.

[7] W. Roh and A. Paulraj, "MIMO channel capacity for the distributed antenna systems," in Proceedings of the IEEE Vehicular Technology Conference (VTC '02), vol. 3, pp. 1520-1524, September 2002.

[8] H. Zhuang, L. Dai, L. Xiao, and Y. Yao, "Spectral efficiency of distributed antenna system with random antenna layout," Electronics Letters, vol. 39, no. 6, pp. 495-496, 2003.

[9] W. Choi and J. G. Andrews, "Downlink performance and capacity of distributed antenna systems in a multicell environment," IEEE Transactions on Wireless Communications, vol. 6, no. 1, pp. 69-73, 2007.

[10] P. M. Shankar, "Outage probabilities of a MIMO scheme in shadowed fading channels with micro- and macrodiversity reception," IEEE Transactions on Wireless Communications, vol. 7, no. 6, pp. 2015-2019, 2008.

[11] P. M. Shankar, "Macrodiversity and microdiversity in correlated shadowed fading channels," IEEE Transactions on Vehicular Technology, vol. 58, no. 2, pp. 727-732, 2009.

[12] S. S. Szyszkowicz, H. Yanikomeroglu, and J. S. Thompson, "On the feasibility of wireless shadowing correlation models," IEEE Transactions on Vehicular Technology, vol. 59, no. 9, pp. 42224236, 2010.

[13] L. Yang, "On the capacity of MIMO Rayleigh fading channels with log-normal shadowing," in Proceedings of the Congress on Image and Signal Processing (CISP '08), vol. 5, pp. 479-482, Sanya, China, May 2008.

[14] L. S. Pillutla and S. K. Jayaweera, "Capacity of MIMO systems in Rayleigh fading and shadowing," in Proceedings of the 
Conference on Signals, Systems and Computers, vol. 1, pp. 11491153, November 2004.

[15] H. Dai, "The impact of shadow fading on the outage capacity and multiuser scheduling gain of MIMO systems," in Proceedings of the International Symposium on Intelligent Signal Processing and Communication Systems (ISPACS '05), pp. 609612, December 2005.

[16] J. D. Sanchez-Heredia, J. F. Valenzuela-Valdés, A. M. MartínezGonzález, and D. A. Sánchez-Hernández, "Emulation of MIMO rician-fading environments with mode-stirred reverberation chambers," IEEE Transactions on Antennas and Propagation, vol. 59, no. 2, pp. 654-660, 2011.

[17] J. F. Valenzuela-Valdés, M. F. Manzano, and L. Landesa, "Evaluation of true polarization diversity in Rician-fading environments," IEEE Antennas and Wireless Propagation Letters, vol. 11, pp. 775-778, 2012.

[18] J. F. Valenzuela-Valdés, M. F. Manzano, and L. Landesa, "Estimating correlation functions for dipoles in correlated Ricianfading scenarios," Journal of Electromagnetic Waves and Applications, vol. 26, no. 16, pp. 2176-2184, 2012.

[19] G. J. Foschini and M. J. Gans, "On limits of wireless communications in a fading environment when using multiple antennas," Wireless Personal Communications, vol. 6, no. 3, pp. 311-335, 1998.

[20] A. Laourine, A. Stéphenne, and S. Affes, "On the capacity of log-normal fading channels," IEEE Transactions on Communications, vol. 57, no. 6, pp. 1603-1607, 2009.

[21] F. Héliot, X. Chu, R. Hoshyar, and R. Tafazolli, "A tight closedform approximation of the log-normal fading channel capacity," IEEE Transactions on Wireless Communications, vol. 8, no. 6, pp. 2842-2847, 2009.

[22] S. Enserink and M. P. Fitz, "Estimation of constrained capacity and outage probability in lognormal channels," IEEE Transactions on Vehicular Technology, vol. 62, no. 1, pp. 399-404, 2013.

[23] X. Zhou, Z. Liu, Z. Wang, H. A. Suraweera, and J. Armstrong, "Capacity analysis for a distributed MIMO-OFDM system in composite spatially correlated channels," in Proceedings of the 2nd International Conference on Communications and Networking in China (ChinaCom '07), pp. 1116-1120, August 2007.

[24] J. Zhang and V. Aalo, "Effect of macrodiversity on averageerror probabilities in a Rician fading channel with correlated lognormal shadowing," IEEE Transactions on Communications, vol. 49, no. 1, pp. 14-18, 2001.

[25] S. H. Tan and T. T. Tjhung, "Error probability of M-ary DPSK in fast Rician fading and lognormal shadowing," Electronics Letters, vol. 32, no. 11, pp. 959-961, 1996.

[26] P. Mary, M. Dohler, J.-M. Gorce, and G. Villemaud, "Symbol error outage analysis of MIMO OSTBC systems over rice fading channels in shadowing environments," IEEE Transactions on Wireless Communications, vol. 10, no. 4, pp. 1009-1014, 2011.

[27] G. Rafiq and M. Pätzold, "The influence of the severity of fading and shadowing on the statistical properties of the capacity of nakagami-lognormal channels," in Proceedings of the IEEE Global Telecommunications Conference (GLOBECOM '08), pp. 1-6, New Orleans, La, USA, December 2008.

[28] L.-C. Wang, W.-C. Liu, and Y.-H. Cheng, "Capacity fades analysis of MIMO Rician channels in mobile ad hoc networks," Performance Evaluation, vol. 66, no. 12, pp. 742-753, 2009.

[29] H. Q. Ngo, E. G. Larsson, and T. L. Marzetta, "Energy and spectral efficiency of very large multiuser MIMO systems," IEEE Transactions on Communications, vol. 61, no. 4, pp. 1436-1449, 2013.
[30] S. Gonzalez-Aurioles, J. F. Valenzuela-Valdés, J. Luis Padilla, P. Padilla, and F. Luna-Valero, "Capacity in weibull fading with shadowing for MIMO distributed system," Wireless Personal Communications, 2014.

[31] F. Rusek, D. Persson, B. K. Lau et al., "Scaling up MIMO: opportunities and challenges with very large arrays," IEEE Signal Processing Magazine, vol. 30, no. 1, pp. 40-60, 2013.

[32] J. F. Valenzuela-Valdês, A. M. Martínez-González, and D. A. Sánchez-Hernández, "Emulation of MIMO nonisotropic fading environments with reverberation chambers," IEEE Antennas and Wireless Propagation Letters, vol. 7, pp. 325-328, 2008.

[33] J. F. Valenzuela-Valdés, A. M. Martínez-González, and D. A. Sánchez-Hernández, "Diversity gain and MIMO capacity for nonisotropic environments using a reverberation chamber," IEEE Antennas and Wireless Propagation Letters, vol. 8, pp. 112$115,2009$. 

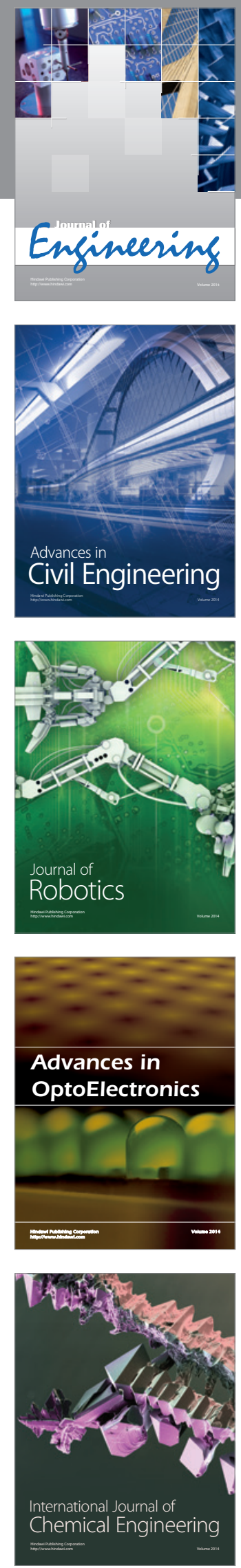

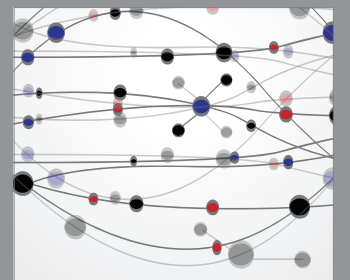

The Scientific World Journal
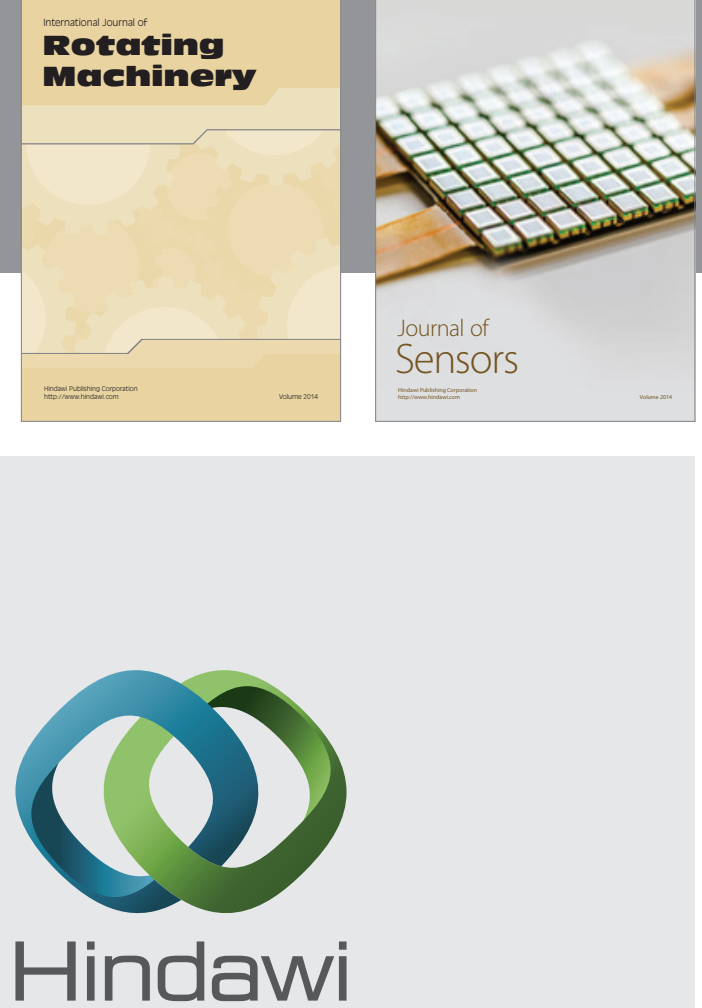

Submit your manuscripts at http://www.hindawi.com
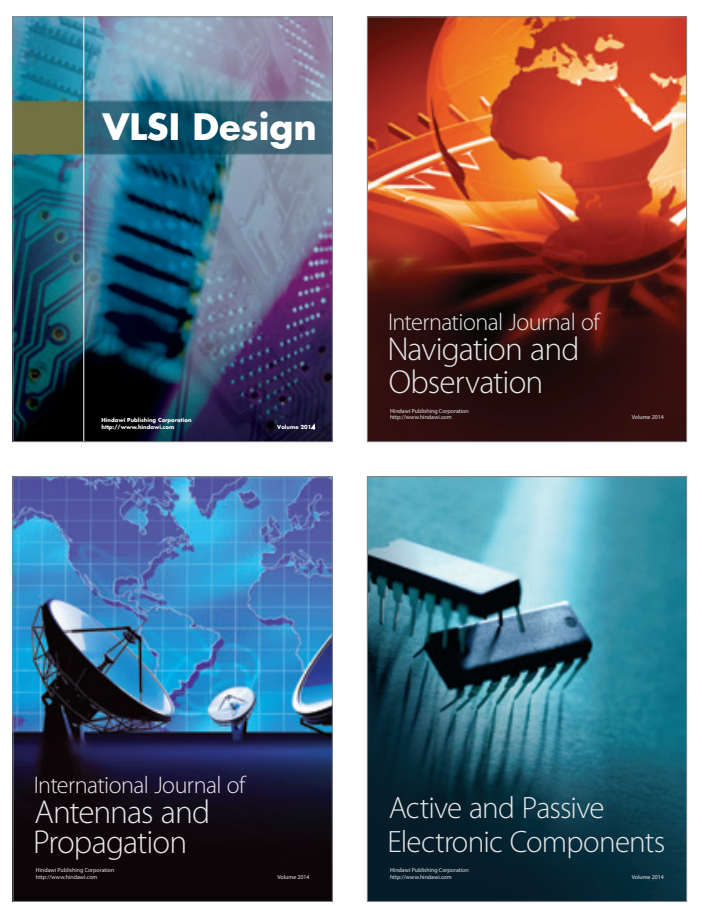
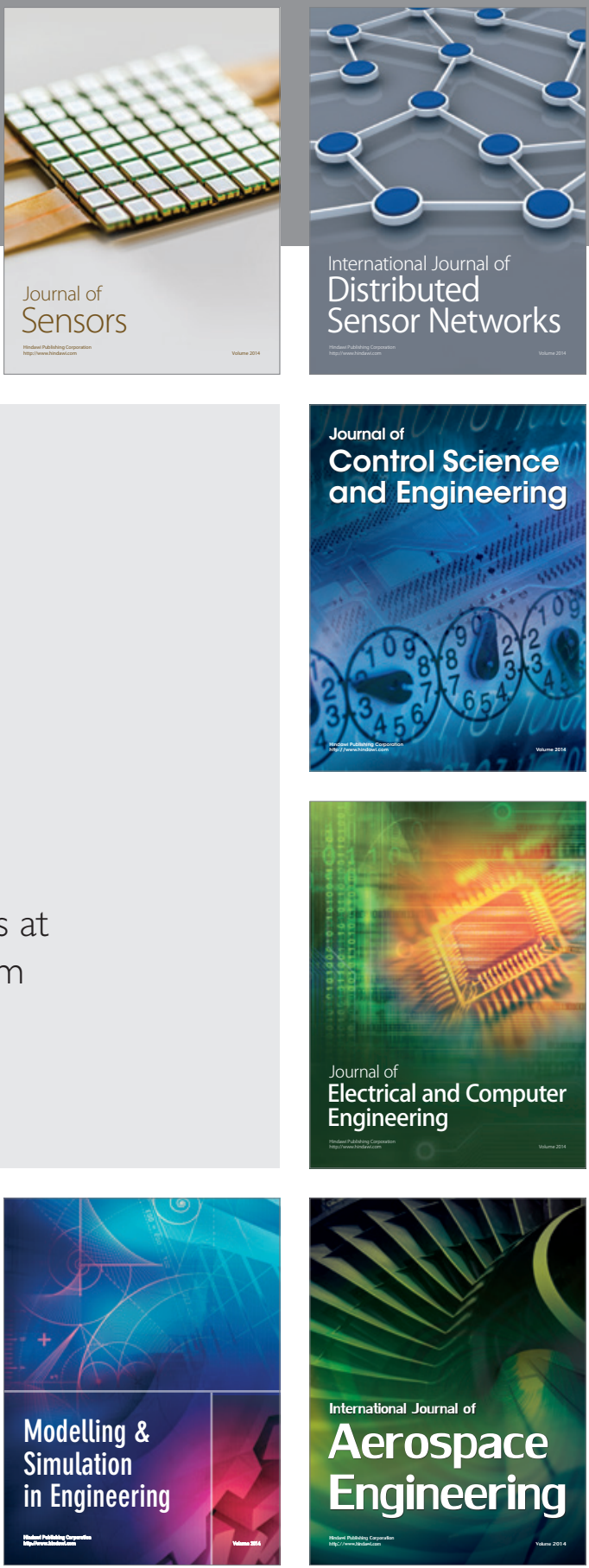

Journal of

Control Science

and Engineering
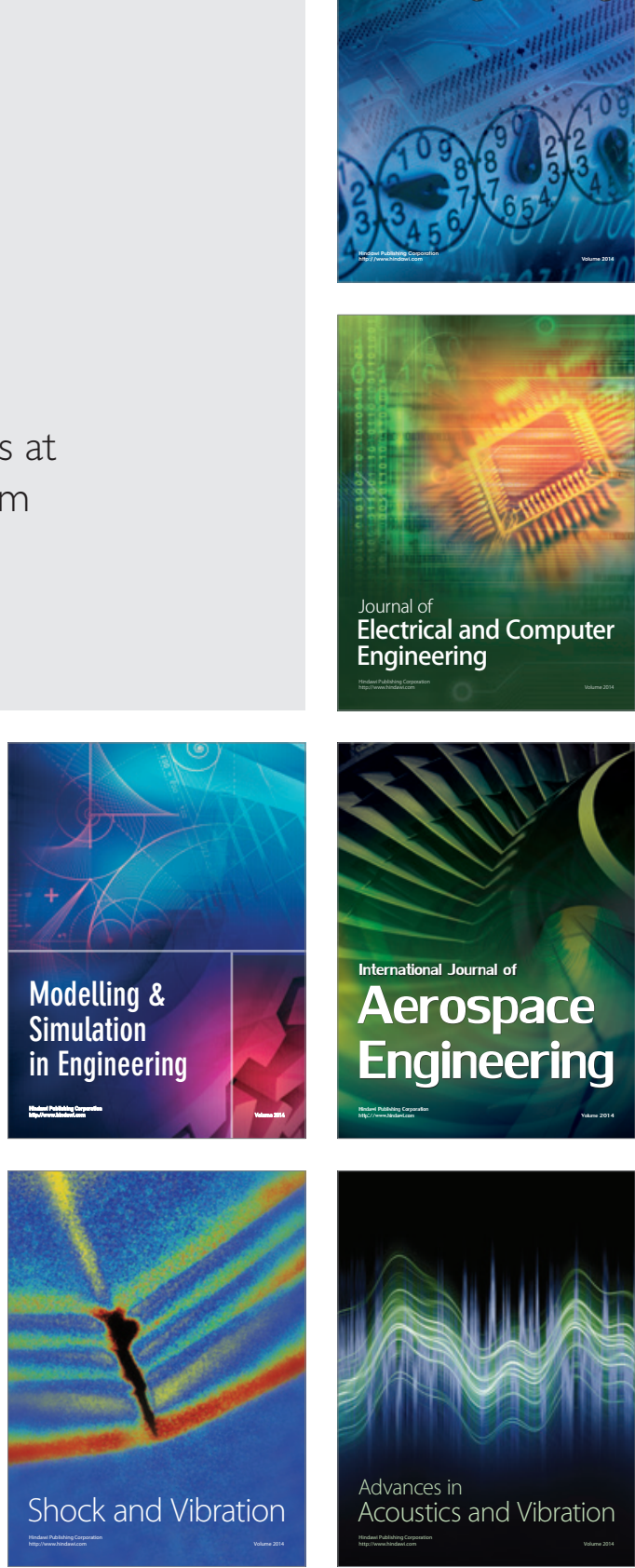